\title{
On $(\delta, \chi)$-bounded families of graphs
}

\author{
András Gyárfás* \\ Computer and Automation Research Institute \\ Hungarian Academy of Sciences \\ Budapest, P.O. Box 63 \\ Budapest, Hungary, H-1518 \\ gyarfas@sztaki.hu
}

\author{
Manouchehr Zaker \\ Department of Mathematics, \\ Institute for Advanced Studies in \\ Basic Sciences (IASBS), \\ Zanjan 45137-66731, Iran \\ mzaker@iasbs.ac.ir
}

Submitted: Jun 7, 2010; Accepted: May 1, 2011; Published: May 8, 2011

Mathematics Subject Classification: 05C15, 05C35

\begin{abstract}
A family $\mathcal{F}$ of graphs is said to be $(\delta, \chi)$-bounded if there exists a function $f(x)$ satisfying $f(x) \rightarrow \infty$ as $x \rightarrow \infty$, such that for any graph $G$ from the family, one has $f(\delta(G)) \leq \chi(G)$, where $\delta(G)$ and $\chi(G)$ denotes the minimum degree and chromatic number of $G$, respectively. Also for any set $\left\{H_{1}, H_{2}, \ldots, H_{k}\right\}$ of graphs by $\operatorname{Forb}\left(H_{1}, H_{2}, \ldots, H_{k}\right)$ we mean the class of graphs that contain no $H_{i}$ as an induced subgraph for any $i=1, \ldots, k$. In this paper we first answer affirmatively the question raised by the second author by showing that for any tree $T$ and positive integer $\ell, F \operatorname{Forb}\left(T, K_{\ell, \ell}\right)$ is a $(\delta, \chi)$-bounded family. Then we obtain a necessary and sufficient condition for $\operatorname{Forb}\left(H_{1}, H_{2}, \ldots, H_{k}\right)$ to be a $(\delta, \chi)$-bounded family, where $\left\{H_{1}, H_{2}, \ldots, H_{k}\right\}$ is any given set of graphs. Next we study $(\delta, \chi)$-boundedness of $\operatorname{Forb}(\mathcal{C})$ where $\mathcal{C}$ is an infinite collection of graphs. We show that for any positive integer $\ell, F \operatorname{Forb}\left(K_{\ell, \ell}, C_{6}, C_{8}, \ldots\right)$ is $(\delta, \chi)$-bounded. Finally we show a similar result when $\mathcal{C}$ is a collection consisting of unicyclic graphs.
\end{abstract}

\section{Introduction}

A family $\mathcal{F}$ of graphs is said to be $(\delta, \chi)$-bounded if there exists a function $f(x)$ satisfying $f(x) \rightarrow \infty$ as $x \rightarrow \infty$, such that for any graph $G$ from the family one has $f(\delta(G)) \leq \chi(G)$, where $\delta(G)$ and $\chi(G)$ denotes the minimum degree and chromatic number of $G$, respectively. Equivalently, the family $\mathcal{F}$ is $(\delta, \chi)$-bounded if $\delta\left(G_{n}\right) \rightarrow \infty$ implies $\chi\left(G_{n}\right) \rightarrow \infty$ for any sequence $G_{1}, G_{2}, \ldots$ with $G_{n} \in \mathcal{F}$. Motivated by Problem 4.3 in [6], the second author introduced and studied $(\delta, \chi)$-bounded families of graphs (under the name of $\delta$-bounded families) in [10]. The so-called color-bound family of graphs mentioned in the related

\footnotetext{
${ }^{*}$ Research supported in part by OTKA Grant No. K68322.
} 
problem of [6] is a family for which there exists a function $f(x)$ satisfying $f(x) \rightarrow \infty$ as $x \rightarrow \infty$, such that for any graph $G$ from the family one has $f(\operatorname{col}(G)) \leq \chi(G)$, where $\operatorname{col}(G)$ is defined as $\operatorname{col}(G)=\max \{\delta(H): H \subseteq G\}+1$. As shown in [10] if we restrict ourselves to hereditary (i.e. closed under taking induced subgraph) families then two concepts $(\delta, \chi)$-bounded and color-bound are equivalent. The first specific results concerning $(\delta, \chi)$-bounded families appeared in [10] where the following theorem was proved (in a somewhat different but equivalent form). In the following theorem for any set $\mathcal{C}$ of graphs, $\operatorname{Forb}(\mathcal{C})$ denotes the class of graphs that contains no member of $\mathcal{C}$ as an induced subgraph.

Theorem 1. ([10]) For any set $\mathcal{C}$ of graphs, Forb $(\mathcal{C})$ is $(\delta, \chi)$-bounded if and only if there exists a constant $c=c(\mathcal{C})$ such that for any bipartite graph $H \in F$ orb $(\mathcal{C})$ one has $\delta(H) \leq c$.

Theorem 1 shows that to decide whether $\operatorname{Forb}(\mathcal{C})$ is $(\delta, \chi)$-bounded we may restrict ourselves to bipartite graphs. We shall make use of this result in proving the following theorems.

Similar to the concept of $(\delta, \chi)$-bounded families is the concept of $\chi$-bounded families. A family $\mathcal{F}$ of graphs is called $\chi$-bounded if for any sequence $G_{i} \in \mathcal{F}$ such that $\chi\left(G_{i}\right) \rightarrow \infty$, it follows that $\omega\left(G_{i}\right) \rightarrow \infty$. The first author conjectured in 1975 [2] (independently by Sumner [9]) the following

Conjecture 1. For any fixed tree T, Forb $(T)$ is $\chi$-bounded.

\section{$2(\delta, \chi)$-bounded families with a finite set of forbidden subgraphs}

The first result in this section shows that for any tree $T$ and positive integer $\ell, F$ orb $\left(T, K_{\ell, \ell}\right)$ is $(\delta, \chi)$-bounded which answers affirmatively a problem of [10].

Theorem 2. For every fixed tree $T$ and fixed integer $\ell$, and for any sequence $G_{i} \in$ Forb $(T$, $\left.K_{\ell, \ell}\right), \delta\left(G_{i}\right) \rightarrow \infty$ implies $\chi\left(G_{i}\right) \rightarrow \infty$.

We shall prove Theorem 2 in the following quantified form.

Theorem 3. For every tree $T$ and for positive integers $\ell, k$ there exist a function $f(T, \ell, k)$ with the following property. If $G$ is a graph with $\delta(G) \geq f(T, \ell, k)$ and $\chi(G) \leq k$ then $G$ contains either $T$ or $K_{\ell, \ell}$ as an induced subgraph.

In Theorem 3 we may assume that the tree $T$ is a complete $p$-ary tree of height $r, T_{p}^{r}$, because these trees contain any tree as an induced subgraph. Using Theorem 1 we note that to prove Theorem 3 it is enough to show the following lemma. 
Lemma 1. For every $p, r, \ell$ there exists $g(p, r, \ell)$ such that the following is true. Every bipartite graph $H$ with $\delta(H) \geq g(p, r, \ell)$ contains either $T_{p}^{r}$ or $K_{\ell, \ell}$ as an induced subgraph.

Proof. To prove the lemma, we prove slightly more. Call a subtree $T \subseteq H$ a distance tree rooted at $v \in V(H)$ if $T$ is rooted at $v$ and for every $w \in V(T)$ the distance of $v$ and $w$ in $T$ is the same as the distance of $v$ and $w$ in $H$. In other words, let $T$ be a subtree of $H$ rooted at $v$ and let $L_{i}$ be the set of vertices at distance $i$ from $v$ in $T$. If $T$ is a distance tree then $L_{i}$ is a subset of the vertices at distance $i$ from $v$ in $H$. Notice that a distance tree $T$ of $H$ is an induced subtree of $H$ if and only if $x y \in E(H)$ implies $x y \in E(T)$ for any $x \in L_{i}, y \in L_{i+1}$. (In this statement it is important that $H$ is a bipartite graph.)

We claim that with a suitable $g(p, r, \ell)$ lower bound for $\delta(H)$, every vertex of a bipartite graph $H$ is the root of an induced distance tree $T_{p}^{r}$ in $H$.

The claim is proved by induction on $r$. For $r=1, g(p, 1, \ell)=p$ is a suitable function for every $\ell, p$. Assuming that $g(p, r, \ell)$ is defined for some $r \geq 1$ and for all $p, \ell$, define $P=p^{r}(\ell-1)$ and

$$
u=g(p, r+1, \ell)=\max \left\{g(P, r, \ell), 1+2^{P p^{r-1}}(\max \{p-1, \ell-1\})\right\}
$$

Suppose that $\delta(H) \geq u, v \in V(H)$. By induction, using that $u \geq g(P, r, \ell)$ by (1), we can find an induced distance tree $T=T_{P}^{r}$ rooted at $v$. In fact we shall only extend a subtree $T^{*}$ of $T$, defined as follows. Keep $p$ from the $P$ subtrees under the root and repeat this at each vertex of the levels $1,2, \ldots r-2$. Finally, at level $r-1$, keep all of the $P$ children at each vertex. Let $L$ denote the set of vertices of $T^{*}$ at level $r, L=\cup_{i=1}^{p^{r-1}} A_{i}$ where the vertices of $A_{i}$ have the same parent in $T^{*},\left|A_{i}\right|=P$. Let $X \subseteq V(H) \backslash V\left(T^{*}\right)$ denote the set of vertices adjacent to some vertex of $L$. (In fact, since $T$ is a distance tree and $H$ is bipartite, $X \subseteq V(H) \backslash V(T)$.) Put the vertices of $X$ into equivalence classes, $x \equiv y$ if and only if $x, y$ are adjacent to the same subset of $L$. There are less than $q=2^{P p^{r-1}}$ equivalence classes (since each vertex of $X$ is adjacent to at least one vertex of $L$ ). Delete from $X$ all vertices of those equivalence classes that are adjacent to at least $\ell$ vertices of $L$. Since $H$ has no $K_{\ell, \ell}$ subgraph, at most $q(\ell-1)$ vertices are deleted. Delete also from $X$ all vertices of those equivalence classes that have at most $p-1$ vertices. During these deletions less than $q(\max \{p-1, \ell-1\})<u-1$ vertices were deleted, the set of remaining vertices is $Y$. It follows from (1) that every vertex of $L$ is adjacent to at least one vertex $y \in Y$, in fact to at least $p$ vertices of $Y$ in the equivalence class of $y$.

Now we plan to select $p$-element sets $\left\{x_{i, 1}, \ldots, x_{i, p}\right\} \subset A_{i}$ and a set $B_{i, j} \subset Y$ of $p$ neighbors of $x_{i, j}$ so that the $B_{i, j}$-S are pairwise disjoint and if $x_{i, j} \in A_{i}$ is adjacent to some $v \in B_{s, t}$ then $s=i, t=j$. Then $\cup_{i=1}^{p^{r-1}} \cup_{j=1}^{p} B_{i, j}$ extends $T^{*}$ to the required induced distance tree $T_{p}^{r+1}$ (there are no edges of $H$ connecting any two $B_{i, j}$-s since $H$ is bipartite).

Start with an arbitrary vertex $x_{1,1} \in A_{1}$. There are at least $p$ neighbors of $x_{1,1}$ in an equivalence class $C$ of $Y$, define $B_{1,1}$ as $p$ elements of $C$. Delete all vertices of $L$ defining $C$ and repeat the procedure. Since at most $(\ell-1)$ vertices are deleted from $L$ at each step, the inequality $\left|A_{i}\right|=P=p^{r}(\ell-1)>\left(p^{r}-1\right)(\ell-1)$ ensures that $\left\{x_{i, j}: 1 \leq i \leq p^{r-1}, 1 \leq j \leq p\right\}$ (and their neighboring sets $B_{i, j}$ ) can be defined. 
Using Theorem 2 we can characterize $(\delta, \chi)$-bounded families of the form $\operatorname{Forb}\left(H_{1}, \ldots, H_{k}\right)$ where $\left\{H_{1}, \ldots, H_{k}\right\}$ is any finite set of graphs. In the following result by a star tree we mean any tree isomorphic to $K_{1, t}$ for some $t \geq 1$.

Corollary 1. Given a finite set of graphs $\left\{H_{1}, H_{2}, \ldots, H_{k}\right\}$. Then Forb $\left(H_{1}, H_{2}, \ldots, H_{k}\right)$ is $(\delta, \chi)$-bounded if and only if one of the following holds:

(i) For some $i, H_{i}$ is a star tree.

(ii) For some $i, H_{i}$ is a forest and for some $j \neq i, H_{j}$ is complete bipartite graph.

Proof. Set for simplicity $\mathcal{F}=F \operatorname{Frb}\left(H_{1}, H_{2}, \ldots, H_{k}\right)$. First assume that $\mathcal{F}$ is $(\delta, \chi)$ bounded. From the well-known fact that for any $d$ and $g$ there are bipartite graphs of minimum degree $d$ and girth $g$, we obtain that some $H_{i}$ should be forest. If $H_{i}$ is star tree then (i) holds. Assume on contrary that none of $H_{i}$ 's is neither star tree nor complete bipartite graph. Then $K_{n, n}$ belongs to $\mathcal{F}$ for some $n$. But this violates the assumption that $\mathcal{F}$ is $(\delta, \chi)$-bounded.

To prove the converse, first note that by a well known fact (see [10]) if $H_{i}$ is a star tree then $\operatorname{Forb}\left(H_{i}\right)$ is $(\delta, \chi)$-bounded. Now since $\mathcal{F} \subseteq \operatorname{Forb}\left(H_{i}\right)$ then $\mathcal{F}$ too is $(\delta, \chi)$-bounded. Now let (ii) hold. We may assume that $H_{i_{0}}$ is forest and $H_{j_{0}}$ is an induced subgraph of $K_{\ell, \ell}$ for some $l$. It is enough to show that $\operatorname{Forb}\left(H_{i_{0}}, K_{\ell, \ell}\right)$ is $(\delta, \chi)$-bounded. If $H_{i_{0}}$ is a tree then the assertion follows by Theorem 2. Let $T_{1}, \ldots, T_{k}$ be the connected components of $H_{i_{0}}$ where $k \geq 2$. We add a new vertex $v$ and connect $v$ to each $T_{i}$ by an edge. The resulting graph is a tree denoted by $T$. We have $\operatorname{Forb}\left(H_{i_{0}}, K_{\ell, \ell}\right) \subseteq \operatorname{Forb}\left(T, K_{\ell, \ell}\right)$ since $H_{i_{0}}$ is induced subgraph of $T$. The proof now completes by applying Theorem 2 for $\operatorname{Forb}\left(T, K_{\ell, \ell}\right)$.

\section{$3(\delta, \chi)$-bounded families with an infinite set of for- bidden subgraphs}

In this section we consider $\operatorname{Forb}\left(H_{1}, H_{2}, \ldots\right)$ where $\left\{H_{1}, H_{2}, \ldots\right\}$ is any infinite collection of graphs. When at least one of the $H_{i}$-s is a tree then the related characterization problem is easy. The following corollary is immediate.

Corollary 2. Let $T$ be any non star tree. Then $\operatorname{Forb}\left(T, H_{1}, \ldots\right)$ is $(\delta, \chi)$-bounded if and only if at least one of $\mathrm{H}_{i}$-s is complete bipartite graph.

When no graph is acyclic in our infinite collection $H_{1}, H_{2}, \ldots$ we are faced with nontrivial problems. The first result in this regard is a result from [8]. They showed that if $G$ is any even-cycle-free graph then $\operatorname{col}(G) \leq 2 \chi(G)+1$. This shows that $\operatorname{Forb}\left(C_{4}, C_{6}, C_{8}, \ldots\right)$ is $(\delta, \chi)$-bounded. Another result concerning even-cycles was obtained in [10] where the following theorem has been proved. Note that $\bar{d}(G)$ stands for the average degree of $G$. 
Theorem 4.([10]) Let $G$ be a graph and $F(G)$ denote the set of all even integers $t$ such that $G$ does not contain any induced cycle of length $t$. Set $A=E \backslash F(G)$ where $E$ is the set of even integers greater than two. Assume that $A=\left\{g_{1}, g_{2}, \ldots\right\}$. Set $\lambda=2 d(d+1)$ where $d=\operatorname{gcd}\left(g_{1}-2, g_{2}-2, \ldots\right)$. If $d \geq 4$ then

$$
\chi(G) \geq \frac{\bar{d}(G)}{\lambda}+1 .
$$

In the following, using a result from [4] we show that for any positive integer $\ell$, $\operatorname{Forb}\left(K_{\ell, \ell}, C_{6}, C_{8}, C_{10}, \ldots\right)$ is $(\delta, \chi)$-bounded. For this purpose we need to introduce bipartite chordal graphs. A bipartite graph $H$ is said to be bipartite chordal if any cycle of length at least 6 in $H$ has at least one chord. Let $H$ be a bipartite graph with bipartition $(X, Y)$. A vertex $v$ of $H$ is simple if for any $u, u^{\prime} \in N(v)$ either $N(u) \subseteq N\left(u^{\prime}\right)$ or $N\left(u^{\prime}\right) \subseteq N(u)$. Suppose that $\mathcal{L}: v_{1}, v_{2}, \ldots, v_{n}$ is a vertex ordering of $H$. For each $i \geq 1$ denote $H\left[v_{i}, v_{i+1}, \ldots, v_{n}\right]$ by $H_{i}$. An ordering $\mathcal{L}$ is said to be a simple elimination ordering of $H$ if $v_{i}$ is a simple vertex in $H_{i}$ for each $i$. The following theorem first appeared in [4] (see also [5]).

Theorem 5. ([4]) Let $H$ be a bipartite graph with bipartition $(X, Y)$. Then $H$ is chordal bipartite if and only if it has a simple elimination ordering. Furthermore, suppose that $H$ is chordal bipartite. Then there is a simple ordering $y_{1}, \ldots, y_{m}, x_{1}, \ldots, x_{n}$ where $X=\left\{x_{1}, \ldots, x_{n}\right\}$ and $Y=\left\{y_{1}, \ldots, y_{m}\right\}$, such that if $x_{i}$ and $x_{k}$ with $i<k$ are both neighbors of some $y_{j}$, then $N_{H^{\prime}}\left(x_{i}\right) \subseteq N_{H^{\prime}}\left(x_{k}\right)$ where $H^{\prime}$ is the subgraph of $H$ induced by $\left\{y_{j}, \ldots, y_{m}, x_{1}, \ldots, x_{n}\right\}$.

In [8] it was shown that $\operatorname{Forb}\left(C_{4}, C_{6}, C_{8}, \ldots\right)$ is $(\delta, \chi)$-bounded. In the following theorem we replace $C_{4}$ by $K_{\ell, \ell}$ for any $\ell \geq 2$.

Theorem 6. Forb $\left(K_{\ell, \ell}, C_{6}, C_{8}, C_{10}, \ldots\right)$ is $(\delta, \chi)$-bounded.

Proof. By Theorem 1 it is enough to show that the minimum degree of any bipartite graph $H \in \operatorname{Forb}\left(K_{\ell, \ell}, C_{6}, C_{8}, C_{10}, \ldots\right)$ is at most $\ell-1$.

Let $H$ be a bipartite $\left(K_{\ell, \ell}, C_{6}, C_{8}, C_{10}, \ldots\right)$-free graph with $\delta(H) \geq \ell$. Let $y_{1}, \ldots, y_{m}, x_{1}$, $\ldots, x_{n}$ be the simple ordering guaranteed by Theorem 5 . Let $d_{H}\left(y_{1}\right)=k$. Note that $k \geq \ell$. The vertex $y_{1}$ has at least $k$ neighbors say $z_{1}, \ldots, z_{k}$ such that $N\left(z_{1}\right) \subseteq N\left(z_{2}\right) \subseteq \ldots \subseteq$ $N\left(z_{k}\right)$. Now since $d_{Y}\left(z_{1}\right) \geq k$, there are $k$ vertices in $Y$ which are all adjacent to $z_{1}$. From the other side $N\left(z_{1}\right) \subseteq N\left(z_{i}\right)$ for any $i=1, \ldots, k$. Therefore all these $k$ neighbors of $z_{1}$ are also adjacent to $z_{i}$ for any $i$. This introduces a subgraph of $H$ isomorphic to $K_{\ell, \ell}$, a contradiction.

We conclude this section with another $(\delta, \chi)$-bounded (infinite) family of graphs. By a unicyclic graph $G$ we mean any connected graph which contains only one cycle. Such a graph is either a cycle or consists of an induced cycle $C$ of length say $i$ and a number of at most $i$ induced subtrees such that each one intersects $C$ in exactly one vertex. We 
call these subtrees (which intersects $C$ in exactly one vertex) the attaching subtrees of $G$. Recall from the previous section that $T_{p}^{r}$ is the $p$-ary tree of height $r$. For any positive integers $p$ and $r$ by a $(p, r)$-unicyclic graph we mean any unicyclic graph whose attaching subtrees are subgraph of $T_{p}^{r}$. We also need to introduce some special instances of unicyclic graphs. For any positive integers $p, r$ and even integer $i$, let us denote the graph consisting of the even cycle $C$ of length $i$ and $i$ vertex disjoint copies of $T_{p}^{r}$ which are attached to the cycle $C$ by $U_{i, p, r}$ (to each vertex of $C$ one copy of $T_{p}^{r}$ is attached).

Proposition 1. For any positive integers $t, p$ and $r$, there exists a constant $c=c(t, p, r)$ such that for any $K_{2, t}$-free bipartite graph $H$ if $\delta(H) \geq c$ then for some even integer $i, H$ contains an induced subgraph isomorphic to $U_{i, p, r}$.

Proof. Let $H$ be any $K_{2, t}$-free bipartite graph. There are two possibilities for the girth $g(H)$ of $H$.

Case 1. $g(H) \geq 4 r+3$. Let $C$ be any smallest cycle in $H$. Since $H$ is bipartite then $C$ has an even length say $i=g(H)$. We prove by induction on $k$ with $0 \leq k \leq i$ that if $\delta(H) \geq g(p, r, t)+2$ then $H$ contains an induced subgraph isomorphic to the graph obtained by $C$ and $k$ attached copies of $T_{p}^{r}$, where $g(p, r, t)$ is as in Lemma 1 . The assertion is trivial for $k=0$. Assume that it is true for $k$ and we prove it for $k+1$. By induction hypothesis we may assume that $H$ contains an induced subgraph $L$ consisting of the cycle $C$ plus $k$ copies of $T_{p}^{r}$ attached to $C$. Let $v$ be a vertex of $C$ at which no tree is attached. Let $e$ and $e^{\prime}$ be two edges on $C$ which are incident with the vertex $v$. We apply Lemma 1 for $H \backslash\left\{e, e^{\prime}\right\}$. Note that since $\delta(H) \geq g(p, r, t)+2$ then the degree of $v$ in $H \backslash\left\{e, e^{\prime}\right\}$ is at least $g(p, r, t)$. We find an induced copy of $T_{p}^{r}$ grown from $v$ in $H \backslash\left\{e, e^{\prime}\right\}$. Denote this copy of $T_{p}^{r}$ by $T_{0}$. Consider the union graph $L \cup T_{0}$. We show that $L \cup T_{0}$ is induced in $H$. We only need to show that no vertex of $T_{0}$ is adjacent to any vertex of $L$. The distance of any vertex in $T_{0}$ from the farthest vertex in $C$ is at most $r+i / 2$. The distance of any vertex in the previous copies of $T_{p}^{r}$ in $L$ from $C$ is at most $r$. Then any two vertices in $T_{0} \cup L$ have distance at most $2 r+i / 2$. Now if there exists an edge between two such vertices we obtain a cycle of length at most $2 r+i / 2+1$ in $H$. By our condition on the girth of $H$ we obtain $2 r+i / 2+1<g(H)$, a contradiction. This proves our induction assertion for $k+1$, in particular the assertion is true for $k=i$. But this means that $H$ contains the cycle $C$ with $i$ copies of $T_{p}^{r}$ attached to $C$ in induced form. The latter subgraph is $U_{i, p, r}$. This completes the proof in this case.

Case 2. $g(H) \leq 4 r+2$. In this case we prove a stronger claim as follows. If $H$ is any $K_{2, t}$-free bipartite graph and $\delta(H) \geq(4 r+2)(t-1)\left(\max \left\{r+1, p^{r+1}\right\}\right)+1$ with $g(H)=i$ then $H$ contains any graph $G$ which is obtained by attaching $k$ trees $T_{1}, \ldots, T_{k}$ to the cycle of length $i$ such that any $T_{j}$ is a subtree of $T_{p}^{r}$ and $k$ is any integer with $0 \leq k \leq i$. It is clear that if we prove this claim then the main assertion is also proved.

Now let $G$ be any graph obtained by the above method. We prove the claim by induction on the order of $G$. If $G$ consists of only a cycle then its length is $i$ and any smallest cycle of $H$ is isomorphic to $G$. Assume now that $G$ contains at least one vertex 
of degree one and let $v$ be any such vertex of $G$. Set $G^{\prime}=G \backslash v$. We may assume that $H$ contains an induced copy of $G^{\prime}$. Denote this copy of $G^{\prime}$ in $H$ by the very $G^{\prime}$. Let $u \in G^{\prime}$ be the neighbor of $v$ in $G$. It is enough to show that there exists a vertex in $H \backslash G^{\prime}$ adjacent to $u$ but not adjacent to any vertex of $G^{\prime}$. Define two subsets as follows: $A=\left\{a \in V\left(G^{\prime}\right): a u \in E\left(G^{\prime}\right)\right\}, \quad B=\left\{b \in V(H) \backslash V\left(G^{\prime}\right): b u \in E(H)\right\}$.

Since $H$ is bipartite and contains no triangle, clearly $A \cup B$ is independent. Let $C=V\left(G^{\prime}\right) \backslash A \backslash\{u\}$. The number of edges between $B$ and $C$ is at most $(t-1)|C|$. We claim that there is a vertex, say $z \in B$, which is not adjacent to any vertex of $C$, since otherwise there will be at least $|B|$ edges between $B$ and $C$. This leads us to $|B| \leq(t-1)|C|$. From other side for the order of $C$ we have $|C| \leq(4 r+2)\left(\max \left\{r+1, p^{r+1}\right\}\right)$. Let $n_{p, r}=(4 r+2)\left(\max \left\{r+1, p^{r+1}\right\}\right)$. We have therefore $|B| \leq(t-1)\left(n_{p, r}-|A|-1\right)$ and $|A|+|B| \leq(t-1) n_{p, r}$. But $|A|+|B|=d(u)>(t-1) n_{p, r}$, a contradiction. Therefore there is a vertex $z$ that is adjacent to $u$ in $H$ but not adjacent to $G^{\prime} \backslash\{u\}$. By adding the edge $u z$ to $G^{\prime}$ we obtain an induced subgraph of $H$ isomorphic to $G$, as desired.

Finally by taking $c=\max \left\{g(p, r, t)+2,(4 r+2)(t-1)\left(\max \left\{r+1, p^{r+1}\right\}\right)+1\right\}$ the proof is completed.

Using Proposition 1 and Theorem 1, we obtain the following result.

Theorem 7. Fix positive integers $t \geq 2, p$ and $r$. For any $i=1,2,3, \ldots$, let $G_{i}$ be any $(p, r)$-unicyclic graph whose cycle has length $2 i+2$. Then Forb $\left(K_{2, t}, G_{1}, G_{2}, \ldots\right)$ is $(\delta, \chi)$-bounded.

\section{Concluding remarks}

If a family $\mathcal{F}$ is both $(\delta, \chi)$-bounded and $\chi$-bounded then it satisfies the following stronger result. For any sequence $G_{1}, G_{2}, \ldots$ with $G_{i} \in \mathcal{F}$ if $\delta\left(G_{i}\right) \rightarrow \infty$ then $\omega\left(G_{i}\right) \rightarrow \infty$. Let us call any family satisfying the latter property, $(\delta, \omega)$-bounded family.

The following result of Rödl (originally unpublished) which was later appeared in Kierstead and Rödl ([7] Theorem 2.3) proves the weaker form of Conjecture 1.

Theorem 8. For every fixed tree $T$ and fixed integer $\ell$, and for any sequence $G_{i} \in \operatorname{Forb}(T$, $\left.K_{\ell, \ell}\right), \chi\left(G_{i}\right) \rightarrow \infty$ implies $\omega\left(G_{i}\right) \rightarrow \infty$.

Combination of Theorem 3 with Theorem 8 shows that $\operatorname{Forb}\left(T, K_{\ell, \ell}\right)$ is $(\delta, \omega)$-bounded.

As we noted before the class of even-hole-free graphs is $(\delta, \chi)$-bounded. It was proved in [1] that if $G$ is even-hole-free graph then $\chi(G) \leq 2 \omega(G)+1$. This implies that Forb $\left(C_{4}, C_{6}, \ldots\right)$ too is $(\delta, \omega)$-bounded.

\section{References}

[1] L. Addario-Berry, M. Chudnovsky, F. Havet, B. Reed, P. Seymour, Bisimplicial vertices in even-hole-free graphs, J. Combin. Theory Ser. B 98 (2008) 1119-1164. 
[2] A. Gyárfás, On Ramsey covering numbers, in Infinite and Finite Sets, Coll. Math. Soc. J. Bolyai, North Holland, New York, 1975, 10, 801-816.

[3] A. Gyárfás, E. Szemerédi, Zs. Tuza, Induced subtrees in graphs of large chromatic number, Discrete Math. 30 (1980) 235-244.

[4] P. L. Hammer, F. Maffray, M. Preissmann, A characterization of chordal bipartite graphs, RUTCOR Research Report, Rutgers University, New Brunswick, NJ, RRR\#16-89, 1989.

[5] J. Huang, Representation characterizations of chordal bipartite graphs, J. Combin. Theory Ser. B 96 (2006) 673-683.

[6] T.R. Jensen, B. Toft, Graph Coloring Problems, Wiley, New York 1995.

[7] H. Kierstead, V. Rödl, Applications of hypergraph coloring to coloring graphs not inducing certain trees, Discrete Math. 150 (1996) 187-193.

[8] S. E. Markossian, G. S. Gasparian, B.A. Reed, $\beta$-perfect graphs, J. Combin. Theory Ser. B 67 (1996) 1-11.

[9] D. P. Sumner, Subtrees of a graph and chromatic number, in: G. Chartrand ed., The Theory and Application of Graphs, Wiley, New York, 1981, 557-576.

[10] M. Zaker, On lower bounds for the chromatic number in terms of vertex degree, Discrete Math., 311 (2011) 1365-1370. 